\title{
Hadamard matrices and strongly regular graphs with the 3-e.c. adjacency property
}

\author{
Anthony Bonato \\ Department of Mathematics, \\ Wilfrid Laurier University, \\ Waterloo, Ontario, Canada, N2L 3C5 \\ abonato@wlu.ca \\ W. H. Holzmann \\ Department of Mathematics and Computer Science, \\ University of Lethbridge, \\ Lethbridge, Alberta, Canada, T1K 3M4 \\ holzmann@uleth.ca \\ Hadi Kharaghani \\ Department of Mathematics and Computer Science, \\ University of Lethbridge, \\ Lethbridge, Alberta, Canada, T1K 3M4 \\ hadi@cs.uleth.ca *
}

Submitted: July 2, 2000; Accepted: November 6, 2000.

\begin{abstract}
A graph is 3-e.c. if for every 3-element subset $S$ of the vertices, and for every subset $T$ of $S$, there is a vertex not in $S$ which is joined to every vertex in $T$ and to no vertex in $S \backslash T$. Although almost all graphs are 3-e.c., the only known examples of strongly regular 3-e.c. graphs are Paley graphs with at least 29 vertices. We construct a new infinite family of 3-e.c. graphs, based on certain Hadamard matrices, that are strongly regular but not Paley graphs. Specifically, we show
\end{abstract}

\footnotetext{
${ }^{*}$ The authors gratefully acknowledge the support of the Natural Sciences and Engineering Research Council of Canada (NSERC)
} 
that Bush-type Hadamard matrices of order $16 n^{2}$ give rise to strongly regular 3 -e.c. graphs, for each odd $n$ for which $4 n$ is the order of a Hadamard matrix.

Key words: $n$-e.c. graphs, strongly regular graphs, adjacency property, Bush-type Hadamard matrix, design

AMS subject classification: Primary 05C50, Secondary 05B20.

\section{Introduction}

Throughout, all graphs are finite and simple. A strongly regular graph $S R G(v, k, \lambda, \mu)$ is a regular graph with $v$ vertices of degree $k$ such that every two joined vertices have exactly $\lambda$ common neighbours, and every two distinct non-joined vertices have exactly $\mu$ common neighbours.

For a fixed integer $n \geq 1$, a graph $G$ is $n$-existentially closed or $n$-e.c. if for every $n$-element subset $S$ of the vertices, and for every subset $T$ of $S$, there is a vertex not in $S$ which is joined to every vertex in $T$ and to no vertex in $S \backslash T$. $N$-e.c. graphs were first studied in [8], where they were called graphs with property $P(n)$. For further background on $n$-e.c. graphs the reader is directed to [5].

If $q$ is a prime power congruent to $1(\bmod 4)$, then the Paley graph of order $q$, written $P_{q}$, is the graph with vertices the elements of $G F(q)$, the field of order $q$, and distinct vertices are joined iff their difference is a square in $\operatorname{GF}(q)$. It is well-known that $P_{q}$ is self-complementary and a $\operatorname{SRG}(q,(q-1) / 2,(q-5) / 4,(q-1) / 4)$. In [1] and [4], it was shown that for a fixed $n$, sufficiently large Paley graphs are $n$-e.c.. Few examples of strongly regular non-Paley $n$-e.c. graphs are known, despite the fact that for a fixed $n$ almost all graphs are $n$-e.c. (see [3] and [9]). The exception is when $n=1$ or 2 ; see [5] and [6]. Even for $n=3$ it has proved difficult to find strongly regular $n$-e.c. graphs that are not Paley graphs. In [1] it was shown that $P_{29}$ is the minimal order 3-e.c. Paley graph. As reported in [5], a 3-e.c. graph has order at least 20, and a computer search has revealed two non-isomorphic 3-e.c. graphs of order 28 , neither of which is strongly regular.

In this article we construct new infinite families of strongly regular 3-e.c. graphs that are not Paley graphs. The graphs we study are constructed from certain Hadamard matrices; in particular, their adjacency matrices correspond to Bush-type Hadamard matrices (see Theorem 5).

A co-clique in a graph is a set of pairwise non-joined vertices. The matrices $I_{n}$ and $J_{n}$ are the $n \times n$ identity matrix and matrix of all ones, respectively. A normalized Hadamard matrix is one whose first row and first column is all ones. For matrices $A, B$, $A \otimes B$ is the tensor or Kronecker product of $A$ and $B$. 


\section{Bush-type Hadamard matrices}

A Hadamard matrix $H$ of order $4 n^{2}$ is called a Bush-type Hadamard matrix if $H=\left[H_{i j}\right]$, where $H_{i j}$ are blocks of order $2 n, H_{i i}=J_{2 n}$ and $H_{i j} J_{2 n}=J_{2 n} H_{i j}$, for $i \neq j, 1 \leq i \leq 2 n$, $1 \leq j \leq 2 n$.

In the language of graphs, a symmetric Bush-type Hadamard matrix of order $4 n^{2}$ is the $\mp$-adjacency matrix ( -1 for adjacency, +1 for non-adjacency) of a strongly regular $\left(4 n^{2}, 2 n^{2}-n, n^{2}-n, n^{2}-n\right)$ graph. See Haemers and Tonchev [10] for a study of such graphs.

K. A. Bush [7] proved that if there exists a projective plane of order $2 n$, then there is a Bush-type Hadamard matrix. Although it is fairly simple to construct Bush-type Hadamard matrices of order $16 n^{2}$, very little is known about the existence or nonexistence of such matrices of order $4 n^{2}$, for $n$ odd. See [11] for details.

For completeness we include the following result of Kharaghani [12].

Theorem 1. If the order of an Hadamard matrix is 4n, then there is a Bush-type Hadamard matrix of order $16 n^{2}$.

Proof. Let $K$ be a normalized Hadamard matrix of order $4 n$. Let $c_{1}, c_{2}, \ldots, c_{4 n}$ be the column vectors of $K$. Let $C_{i}=c_{i} c_{i}^{t}$, for $i=1,2, \ldots, 4 n$. Then it is easy to see that:

1. $C_{i}^{t}=C_{i}$, for $i=1,2, \ldots, 4 n$;

2. $C_{1}=J_{4 n}, C_{i} J_{4 n}=J_{4 n} C_{i}=0$, for $i=2,3, \ldots, 4 n$;

3. $C_{i} C_{j}^{t}=0$, for $i \neq j, 1 \leq i, j \leq n$;

4.

$$
\sum_{i=1}^{4 n} C_{i} C_{i}^{t}=16 n^{2} I_{4 n}
$$

Now consider a symmetric Latin square with entries $1,2, \ldots, 4 n$ with constant diagonal 1. Replace each $i$ by $C_{i}$. We then obtain a Bush-type Hadamard matrix of order $16 n^{2}$.

Example 2. We give an example of a Bush-type Hadamard matrix of order 64. For ease of notation, we use - instead of -1 . Let $K$ be the following Hadamard matrix:

$$
\begin{aligned}
& \left(\begin{array}{cccccccc}
1 & 1 & 1 & 1 & 1 & 1 & 1 & 1 \\
1 & - & 1 & - & 1 & - & 1 & - \\
1 & 1 & - & - & 1 & 1 & - & - \\
1 & - & - & 1 & 1 & - & - & 1 \\
1 & 1 & 1 & 1 & - & - & - & - \\
1 & - & 1 & - & - & 1 & - & 1 \\
1 & 1 & - & - & - & - & 1 & 1 \\
1 & - & - & 1 & - & 1 & 1 & -
\end{array}\right) \\
= & \left(\begin{array}{llllllll}
c_{1} & c_{2} & c_{3} & c_{4} & c_{5} & c_{6} & c_{7} & c_{8}
\end{array}\right)
\end{aligned}
$$

THE ELECTRONIC JOURNAL OF COMBINATORICS 8 (2001), \#R1 
Then for $i=1, \ldots, 8$, let

$$
C_{i}=c_{i} c_{i}^{t},
$$

and let

$$
H=\left(\begin{array}{llllllll}
C_{1} & C_{2} & C_{3} & C_{4} & C_{5} & C_{6} & C_{7} & C_{8} \\
C_{2} & C_{1} & C_{4} & C_{3} & C_{6} & C_{5} & C_{8} & C_{7} \\
C_{3} & C_{4} & C_{1} & C_{2} & C_{7} & C_{8} & C_{5} & C_{6} \\
C_{4} & C_{3} & C_{2} & C_{1} & C_{8} & C_{7} & C_{6} & C_{5} \\
C_{5} & C_{6} & C_{7} & C_{8} & C_{1} & C_{2} & C_{3} & C_{4} \\
C_{6} & C_{5} & C_{8} & C_{7} & C_{2} & C_{1} & C_{4} & C_{3} \\
C_{7} & C_{8} & C_{5} & C_{6} & C_{3} & C_{4} & C_{1} & C_{2} \\
C_{8} & C_{7} & C_{6} & C_{5} & C_{4} & C_{3} & C_{2} & C_{1}
\end{array}\right)
$$

By Theorem 1, $H$ is a Bush-type Hadamard matrix of order 64 .

Lemma 3. Let $H=\left[H_{i j}\right]$ be a Bush-type Hadamard matrix of order $4 n^{2}$. Let $M=$ $H-I_{2 n} \otimes J_{2 n}$. Then $M$ contains two $S R G\left(4 n^{2}, 2 n^{2}-n, n^{2}-n, n^{2}-n\right)$.

Proof. The row sums of $H$ are all $2 n$. Thus the negative entries in $H$ can be viewed as the incidence matrix of a $S R G\left(4 n^{2}, 2 n^{2}-n, n^{2}-n, n^{2}-n\right)$-graph. Since negating all the off diagonal blocks of $H$ leaves a Bush-type Hadamard, the positive entries of $M$ also form a $S R G\left(4 n^{2}, 2 n^{2}-n, n^{2}-n, n^{2}-n\right)$-graph.

Note that the two graphs may not be isomorphic in general. We call the matrix $M$ a twin graph.

\section{Bush-type Hadamard matrices and 3-e.c. graphs}

A graph $G$ is 3-e.c. if for each triple $x, y, z$ of distinct vertices from $G$, there are 8 vertices from $V(G) \backslash\{x, y, z\}$, one joined to each of $x, y, z ; 3$ joined to exactly two of $x, y, z ; 3$ joined to exactly one of $x, y, z$; and one joined to none of $x, y, z$. From the perspective of the $(1,-1)$-adjacency matrix $A$ of $G$ this is equivalent to the following condition: for each 3 distinct rows $r_{1}, r_{2}, r_{3}$ from $A$, representing vertices $x, y, z$ in $G$, there are 8 columns in the submatrix formed by $r_{1}, r_{2}, r_{3}$, distinct from the columns representing $x, y, z$, which contain all the 8 possible patterns of 1's and -1 's. Since our graphs are constructed as $(1,-1)$-adjacency matrices, we use the latter condition when checking whether our graphs are 3-e.c.. We first prove the following lemma.

Lemma 4. Let $K$ be a normalized Hadamard matrix of order $4 n$ with $n$ odd and $n>1$. Consider any $3 \times n$ submatrix of $K$ not including the first row of $K$. Each of the eight possible sign patterns appears as a column at least once among the columns of the submatrix. 
Proof. Let $K$ be a normalized Hadamard matrix of order $4 n$. Consider any three rows of $K$ which do not include the first row. Without loss of generality, we may assume that the rows have the form

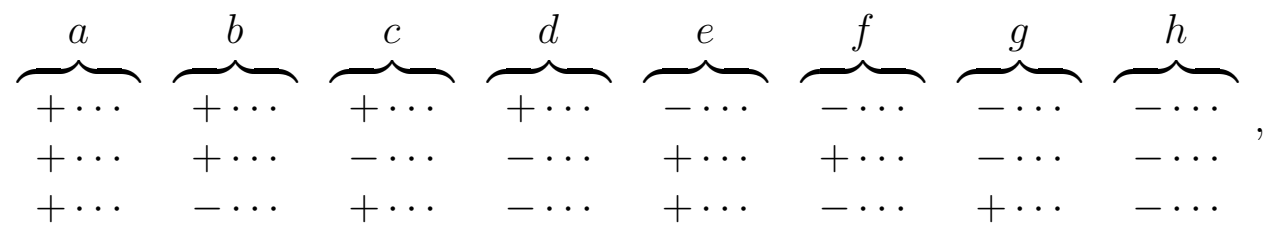

where each letter is a nonnegative integer. This leads to the linear system

$$
\begin{aligned}
a+b+c+d+e+f+g+h & =4 n \\
a+b+c+d & =2 n \\
a+b+e+f & =2 n \\
a+c+e+g & =2 n \\
a+b-c-d-e-f+g+h & =0 \\
a-b+c-d-e+f-g+h & =0 \\
a-b-c+d+e-f-g+h & =0
\end{aligned}
$$

It can be seen that the solution for this system is $b=c=e=h$ and $a=d=f=$ $g=n-h$.

We need to find a positive solution to the system. Since $K$ is normalized, $a>0$ so $h \neq n$. It is enough to show that $h=0$ is not possible. If $h=0$, then the three selected rows have the following form:

$$
\begin{array}{llll}
\overbrace{+\cdots}^{a=n} & \overbrace{+\cdots}^{d=n} & \overbrace{-\cdots}^{f=n} & \overbrace{-\cdots}^{g=n} \\
+\cdots & -\cdots & +\cdots & -\cdots \\
+\cdots & -\cdots & -\cdots & +\cdots
\end{array}
$$

Now consider a fourth row, and without loss of generality, we can rearrange the columns so that the rows have the form:

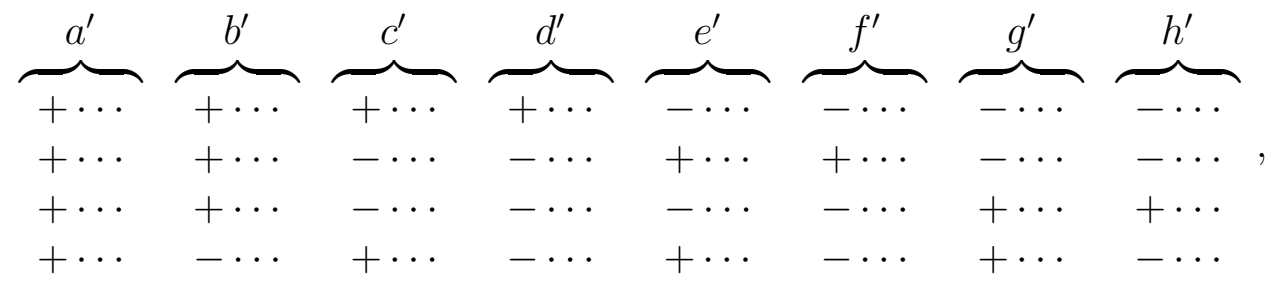

where each primed letter is a nonnegative integer. 
This leads to the system

$$
\begin{aligned}
a^{\prime}+b^{\prime} & =n \\
c^{\prime}+d^{\prime} & =n \\
e^{\prime}+f^{\prime} & =n \\
g^{\prime}+h^{\prime} & =n \\
a^{\prime}-b^{\prime}+c^{\prime}-d^{\prime}-e^{\prime}+f^{\prime}-g^{\prime}+h^{\prime} & =0 \\
a^{\prime}-b^{\prime}-c^{\prime}+d^{\prime}-e^{\prime}+f^{\prime}+g^{\prime}-h^{\prime} & =0 \\
a^{\prime}-b^{\prime}-c^{\prime}+d^{\prime}+e^{\prime}-f^{\prime}-g^{\prime}+h^{\prime} & =0 \\
a^{\prime}+c^{\prime}+e^{\prime}+g^{\prime} & =2 n
\end{aligned}
$$

whose solution is $a^{\prime}=b^{\prime}=c^{\prime}=d^{\prime}=e^{\prime}=f^{\prime}=g^{\prime}=h^{\prime}=n / 2$. Thus $n$ must be even, a contradiction.

Theorem 5. Let $4 n$ be the order of a Hadamard matrix, $n$ odd, $n>1$. There is a Bush-type Hadamard matrix of order $16 n^{2}$ which is the adjacency matrix of a twin $S R G\left(16 n^{2}, 8 n^{2}-2 n, 4 n^{2}-2 n, 4 n^{2}-2 n\right)$, whose vertices can be partitioned into $4 n$ disjoint co-cliques of order $4 n$. Furthermore, the graph is 3-e.c..

Proof. Consider the Bush-type Hadamard matrix $H=\left[H_{i j}\right]$, where $H_{i j}$ is the $i j$ block of $H$ of size $4 n \times 4 n$, constructed in Theorem 1 from a Hadamard matrix of order $4 n$. The fact that there is a twin $S R G\left(16 n^{2}, 8 n^{2}-2 n, 4 n^{2}-2 n, 4 n^{2}-2 n\right)$ whose vertices can be partitioned into $4 n$ disjoint co-cliques of order $4 n$, follows from Lemma 3.

It remains to show that the graph is 3-e.c.. Given three rows of $H$, consider the submatrix $L$ consisting of these three rows. We need to show that each of the eight possible sign patterns appears as a column among the columns of the submatrix.

The rows of $H$ can be partitioned into $4 n$ "zones", corresponding to the rows of the $4 n 4 n \times 4 n$ subblocks of $H$. We consider three cases, based on where the three rows of $L$ are located relative to the zones.

Case 1: The rows of $L$ are selected from the same zone, say the $j$-th zone. Referring to the proof of Theorem 1 we see that the leading columns of $C_{i}$ 's form a rearrangement of the columns of the original Hadamard matrix. The only case when not all 8 patterns appear among the leading columns is if the leading columns appear in form like in matrix (1). However, all $C_{i}$ 's are of rank 1, so the negatives of each of the patterns in the columns of matrix (1) appear among the columns of $H$, off the block $H_{j j}$. Thus all eight patterns appear off the block $H_{j j}$.

Case 2: Exactly two rows of $L$ belong to the same zone. Suppose two rows $r_{1}$ and $r_{2}$ are from the $j$-th zone and the other row, $r_{3}$, is from the $k$-th zone, where $k \neq j$. Without loss of generality we can assume that the entries of the blocks in the $r_{1}$ and $r_{2}$ 
rows have the form:

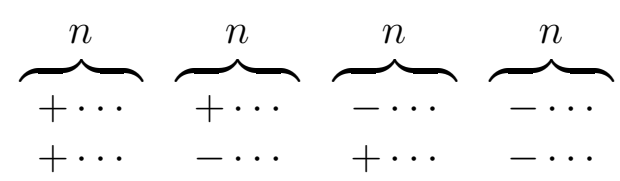

We now look at the possible arrangement of row $r_{1}$ relative to row $r_{3}$. We observe that in each block a similar arrangement as in (2) occurs. Since in each block, row $r_{2}$ is a multiple of row $r_{1}$, we see that all eight patterns appear off the blocks $H_{j j}$ and $H_{k k}$.

Case 3: The three rows of $L$ belong to three different zones, zones $i, j$, and $k$, with $i, j$, and $k$ distinct. Select $l$ distinct from $i, j$, and $k$. Consider the three rows restricted to the blocks $H_{i l}, H_{j l}$, and $H_{k l}$. The rows are multiples of three distinct rows of the original Hadamard matrix, so by Lemma 4 all eight patterns appear off the blocks $H_{i i}$, $H_{j j}$, and $H_{k k}$. (Note that the assumption that $n$ is odd is only used in this part of the proof.)

Of course, none of the graphs in Theorem 5 are Paley graphs. We think that the assumption that $n$ is odd can be dropped from Theorem 5, in view of the following example and remark, and the proof above.

Example 6. A Bush-type Hadamard matrix of order 64 is not included in the previous theorem. However, Example 2 leads to two (isomorphic) graphs which were verified to be 3-e.c. by a computer calculation. We have verified that this graph is not 4-e.c.. We do not know an example of a 4-e.c. graph that is not a Paley graph.

Remark 7. The only known Bush-type Hadamard matrix of order $4 n^{2}, n$ odd, $n>1$ is of order 324 and is constructed in [11]. We tested this Bush-type Hadamard matrix of order 324 by computer and have established that its graph is 3-e.c..

These observations lead us to the following conjecture.

Conjecture 8. Every Bush-type Hadamard matrix of order $4 n^{2}$ with $n>1$ contains a twin 3-e.c. $S R G\left(4 n^{2}, 2 n^{2}-n, n^{2}-n, n^{2}-n\right)$.

We are grateful to the referee for pointing out a few minor errors and the following. There are some strongly regular 3-e.c. graphs that are not Paley graphs. Some of them, however, do have the same parameters. For example, there are 3-e.c. graphs that are not Paley graphs, but have the same parameters as $P_{37}, P_{41}$, and $P_{49}$. Furthermore, although there does not exist a symmetric Bush-type Hadamard matrix of order 36 (see for example [2]), there is a unique 3-e.c. $(36,15,6,6)$ graph which is reproduced in Figure 1. 
[ 011111111111111100000000000000000000 101111110000000011111111000000000000 110111101000000010000000111111100000 111010010110000001100000111000011100 111100000101100001011000100110000011 111000011011000000000111000101110010 111000010000011110110000000011101001 110101100000011000000110100000011111 101001000001011111000001011100000110 100110000011100110000101010010011001 100101000101100100110010001001110100 100011001110010000101001000011000111 100010000110011100011110110100100000 100000111001101000101010010110001100 100000111000110101001001101000110001 100000101110101011010100001001001010 011000101100000100010111011010000101 010110001000001100111001001100011010 010100100011010001011001010001101100 010010100010100111100010000100100111 010010000001111001100111101011000000 010001010100100110001011110001001010 010001010010110010011100001110010100 010001001101001011101100010000110001 001110010000101000001100011001100111 001100001100110010100101100100101100 001100001010001111001010100011010100 001011001000110001010010010010111010 001010100101010010001010001101011001 001001100011000100101100101010101010 001001100010101000110001110101010001 000101010110001001000011001110101001 000100110100010101100100010111010010 000100011011010010110010111000000011 000011011001000101010100100101001101 000010110101001010010001100010110110 .

Figure 1: A 3-e.c. $(36,15,6,6)$ graph 


\section{References}

[1] W. Ananchuen and L. Caccetta, On the adjacency properties of Paley graphs, Networks 23 (1993), 227-236.

[2] F. C. Bussemaker, W. H. Haemers and E. Spence, The Search for Pseudo Orthogonal Latin Squares of order 6, Des., Codes Crytogr., to appear.

[3] A. Blass and F. Harary, Properties of almost all graphs and complexes, J. Graph Theory 3 (1979), 225-240.

[4] A. Blass, G. Exoo, and F. Harary, Paley graphs satisfy all first-order adjacency axioms, J. Graph Theory 5 (1981), no. 4, 435-439.

[5] A. Bonato, K. Cameron, On an adjacency property of almost all graphs, to appear.

[6] A. Bonato, K. Cameron, On 2-e.c. line-critical graphs, to appear.

[7] K. A. Bush, Unbalanced Hadamard matrices and finite projective planes of even order, J. Combin. Theory 11 (1971), 38-44.

[8] L. Caccetta, L., P. Erdős, and K. Vijayan, A property of random graphs, Ars Combin. 19 (1985), 287-294.

[9] R. Fagin, Probabilities on finite models, J. Symbolic Logic 41 (1976), 50-58.

[10] W. H. Haemers and V. D. Tonchev, Spreads in strongly regular graphs, Des. Codes Cryptogr. 8 (1996), 145-157.

[11] Z. Janko, H. Kharaghani and V. D. Tonchev, The existence of a Bush-type Hadamard matrix of order 324 and two new infinite classes of symmetric designs, Des. Codes Cryptogr., to appear.

[12] H. Kharaghani, New classes of weighing matrices, Ars Combin. 19 (1985), 69-72.

[13] H. Kharaghani, On the twin designs with the Ionin-type parameters, Electron. J. Combin. 7 (2000), \#R1. 